\title{
Unresolved Pre-Main Sequence Binaries in the HR Diagram
}

\author{
Patrick Hartigan \\ Department of Physics and Astronomy, Rice University, 6100 S. Main, \\ Houston TX 77005-1892 \\ Scott Kenyon \\ Center for Astrophysics, Mail Stop 16, 60 Garden St. Cambridge MA \\ 02138
}

\begin{abstract}
We consider how positions of unresolved pre-main-sequence binaries in the HR diagram compare with the positions of their corresponding primaries and secondaries. As one might expect from simple considerations, the composites appear younger than their components, and have masses intermediate between those of the two stars. However, the amount of the age difference between the composite and its components ranges from nearly zero to as much as an order of magnitude depending upon the specifics of the system such as the reddening and relative spectral types of the objects. We present four examples that illustrate these issues.
\end{abstract}

\section{Introduction}

Most studies of star formation rely in some way upon estimates of pre-mainsequence stellar masses and ages. These estimates are usually done by comparing the location of a star in the HR diagram, determined by its spectral type and luminosity, with a set of theoretical masses and isochrones. However, most young stars are binaries, and the majority of these have separations less than $100 \mathrm{AU}$ (e.g., Ghez, Neugebauer \& Matthews 1993). At the distance to the nearest populous regions of star formation (150 AU - 450 AU), such pairs have subarcsecond separations and will be unresolved in typical ground-based observations. The question naturally arises as to how the mass and age of an unresolved system compares with the masses and ages of the primary and secondary.

At first glance the problem may seem rather trivial. If a star once thought to be single is resolved into two identical components, the spectral types of the components, and hence their effective temperatures, will stay the same as that of the composite. Because mass tracks are nearly vertical for young low mass stars in the HR diagram, each component will have a mass close to that inferred for the composite. The luminosity of each component will be half that of the composite, or 0.3 lower in $\log \mathrm{L}$, which makes the component stars appear older than the composite. The magnitude of the age shift depends on the theoretical tracks used and the location of the star in the HR diagram. The other limiting case, 
that of a faint secondary around a bright primary, results in a composite age and mass close to that of the primary because the secondary does not significantly affect the composite spectrum or luminosity.

However, away from these limiting cases the situation is less clear. For example, an M2 companion to a $\mathrm{K} 5$ star may add enough $\mathrm{TiO}$ absorption to move the composite spectrum to an intermediate spectral type like M0. In such a case the inferred luminosity of the composite will be affected both by a different reddening correction, and by a new bolometric correction. Both corrections can have a marked influence on the luminosity estimate and therefore the age estimate. Indeed, comparisons of composite spectra of unresolved binaries with those of the resolved components sometimes show a composite with a luminosity as much as 3 times that of the brighter component (Brandner \& Zinnecker 1997).

In this paper we compare the HR diagram location of several subarcsecond pre-main-sequence binaries observed with HST with the location of a composite generated from the sum of each component and reduced as if the composite were a single star. The effect of duplicity on the placement of stars in the HR diagram is generally what one would expect from the simple considerations outlined above, though complications can occur when the spectral types of primary and secondary differ markedly and the secondary adds a sufficient fraction of the total flux. The following uses the DM97 pre-main-sequence tracks (D'Antona \& Mazzitelli 1997), but the results are qualitatively the same for all such curves (e.g. Baraffe et al. 1998).

\section{Results}

\subsection{Case 1: Equal Components}

The first case we consider is FO Tau, a binary with a separation of $0.17^{\prime \prime}$ (24 AU). The component stars have nearly identical veiled M3.5 spectra, with similar reddening. The primary has a somewhat stronger $\mathrm{H} \alpha$ emission line, as well as bright [O I] 6300 and IR Ca II triplet emission which is lacking in the secondary, but the spectral types of each star are identical to that of a composite formed from their sum. As discussed above, the mass of the each component is the same as that of the composite, but the the primary and secondary are older than the composite by about an order of magnitude for the DM97 tracks $\left(7 \times 10^{5} \mathrm{yr}\right.$ as compared with $7 \times 10^{4} \mathrm{yr}$; Fig. 1) This age difference would drop to a factor of $\sim 2$ if the stars were about a factor of three less luminous because for these tracks the isochrones are particularly close together around $3 \times 10^{5} \mathrm{yr}$ for 0.2 $\mathrm{M}_{\odot}$ stars.

For the simple case of Kelvin-Helmholtz contraction at constant $\mathrm{T}_{e}$, the age $\tau \sim \mathrm{GM}^{2} / \mathrm{RL} \sim \mathrm{L}^{-1.5}$ (using $\mathrm{L} \sim \mathrm{R}^{2} \mathrm{~T}_{e}^{4}$ ). Hence, $\Delta \log (\tau)=-1.5 \Delta \log (\mathrm{L})$. For equal component binaries the age difference between the composite and the components is 0.45 in $\log (\tau)$, which is a factor of 2.8 in age.

\subsection{Case 2: Different Components, Faint Secondary}

Fig. 1 shows that the composite spectrum of UY Aur lies very close to that of the primary, even though the secondary contributes $39 \%$ of the composite luminosity and has a markedly later spectral type (M2.5) than that of the primary (K7). 


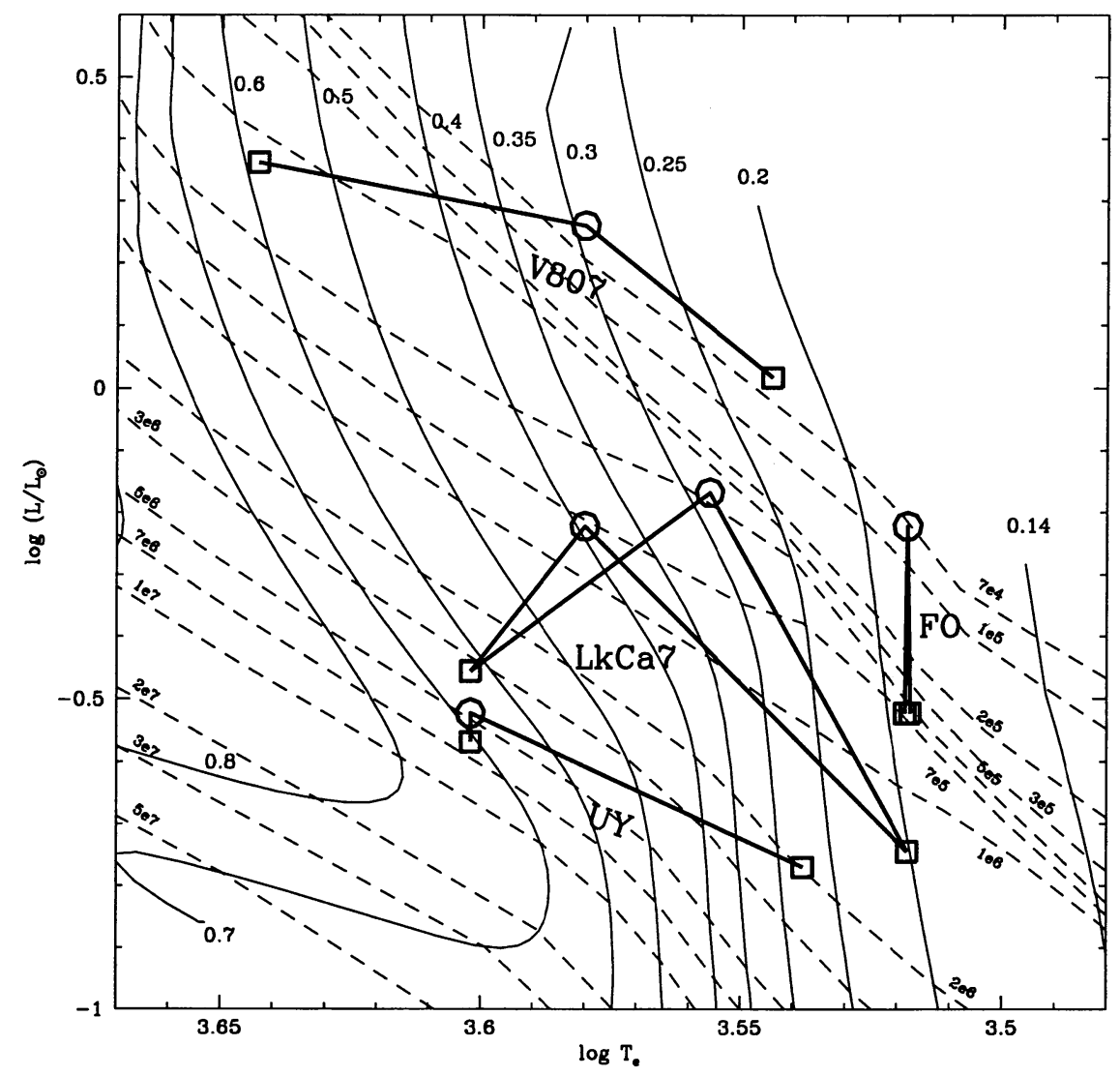

Figure 1. The resolved components (squares) and composites (circles) of several subarcsecond pre-main-sequence binaries in the HR diagram as determined from HST spectra (except for the V807 composite, which is from HSS). In each pair the primary and secondary are connected to the composite by a heavy line. Theoretical tracks are from the 002.d25 models of DM97; solid curves are mass tracks in $\mathrm{M}_{\odot}$, and the dashed lines are isochrones in years. The amount that the composite differs from the primary varies between the pairs. The two composites for $\mathrm{Lk} \mathrm{Ca} 7$ are discussed in the text. 
The reason that the secondary is essentially irrelevant to the composite is that the secondary is much more reddened than the primary $\left(\mathrm{A}_{V}=2.65\right.$ as compared with 0.65). Hence, the observed flux of the secondary is much smaller than that of the primary, and hardly affects the composite spectrum even though the secondary has a substantial luminosity once it has been dereddened. As Koresko noted in his review talk (see also Herbst, Koresko \& Leinert 1995), the secondary of UY Aur has been known since the first observations of Joy \& Biesbrock (1944) to have a highly variable reddening, so observations of a composite UY Aur spectrum taken at different times should show spectral variability depending on how much the secondary is obscured.

\subsection{Case 3: Different Components, Bright Secondary}

LkCa7 consists of a pair of wTTs with quite different spectral types, K7 and M3.5. As in UY Aur, the secondary in this system contributes a significant fraction $(34 \%)$ of the composite luminosity. However, unlike UY Aur, the reddening to each component is similar in LkCa7. Because the secondary is much redder than the primary, the composite spectrum looks later ( M1 - M1.5) longward of $\sim 8000 \AA$, and much bluer $(\sim \mathrm{M} 0)$ around $6000 \AA$. Here the secondary contributes enough flux to the composite that its $\mathrm{TiO}$ bands become noticeable, which makes the composite spectral type later.

The composite luminosity is $0.68 \mathrm{~L}_{\odot}$ for a $\mathrm{M} 1.5$ spectrum and $0.60 \mathrm{~L}_{\odot}$ for the $\mathrm{M} 0$ case (Fig. 1). Both values are actually larger than $0.53 \mathrm{~L}_{\odot}$, the sum of the luminosities of the individual components. The reason for this result is that both bolometric corrections and reddenings are affected by the choice of the composite spectral type, and together these influence the final luminosity estimate. In this case, the mass of the composite lies roughly midway between that of the primary and secondary, and has an age about a factor of 5 lower than that of the primary. Hence, even though the location in the HR diagram of the primary and secondary of LkCa7 resemble those of UY Aur, the composite spectra look quite different in the two systems because of the reddening differences between the UY Aur primary and secondary.

\subsection{Case 4: Different Components, Differing Reddening Procedures}

The final binary we consider is V 807 Tau, a very young wTTs pair with markedly differing spectral types for the primary and secondary (K5 \& M2, respectively). To highlight how different methods affect the results, in this case we take the location of the composite to be that of Hartigan, Strom, \& Strom (1994; hereafter HSS), who obtained the effective temperature from spectra, but determined the reddening from color excesses rather than from the spectra as we have done elsewhere in this paper. As Fig. 1 shows, the location of the HSS composite in this case lies near the line that connects the primary and secondary, and actually has a luminosity less than that of the primary.

The reason for this odd behavior is again the reddening. The presence of a companion significantly alters the intrinsic stellar colors of the system, in this case making the system appear redder as well as making the composite spectral type later than that of the primary. The result is an $A_{V}$ of 0.0 if one uses the composite spectrum and the observed colors. The actual visual reddening of each component measured from the HST spectra is about 0.5 magnitudes. 
If this reddening correction is applied to the composite spectrum the inferred luminosity rises well above that of the primary alone, much as it does in LkCa7.

\section{Summary}

As expected, an unresolved low mass pre-main-sequence binary generally appears more luminous, and therefore younger than its component stars. The mass inferred from the composite will lie somewhere between that of the primary and secondary. However, the factor by which the age decreases in the composite and the actual location of the composite in the HR diagram depends upon several factors, such as the reddening, relative stellar fluxes at the wavelengths that the stars are classified, and the differences in the spectral types of the components. The resulting age differences between the composite and its components can range anywhere from nearly zero to as much as an order of magnitude, with the simple case of two equal pairs and vertical evolutionary tracks giving a factor of 2.8 in age. These complexities will make it difficult to correct for unresolved binaries in more than an approximate manner, for example, in surveys of the initial mass functions of young clusters.

\section{References}

Baraffe, I., Chabrier, G., Allard, F., \& Hauschildt, P. H. 1998, A\&A, 337, 403 Brandner, W., \& Zinnecker, H. 1997, A\&A, 321, 220

D'Antona, F., \& Mazzitelli, I. 1997, Evolution of Low Mass Stars in Cool Stars in Clusters and Associations, ed. R. Pallavicini \& G. Micela, Mem. S. A. It., 68,807

Ghez, A., Neugebauer, G., \& Matthews, K. 1993, AJ, 106, 2005.

Hartigan, P., Strom, K. M., \& Strom, S. E. 1994, ApJ, 427, 961.

Herbst, T. M., Koresko, C. D., \& Leinert, C. 1995, ApJ, 444, L93.

Joy, A. H., \& van Biesbrock, G. 1944, PASP, 56, 119.

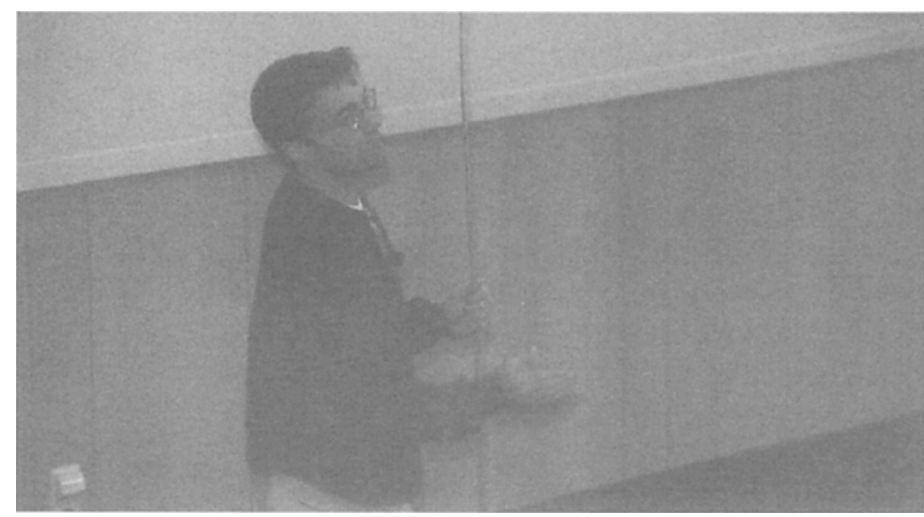

Pat Hartigan 